\title{
The Technical Evolution of CA Storage Protocols and the Advancements in Elucidating the Fruit Responses to Low Oxygen Stress
}

\author{
P. Tonutti \\ Institute of Life Sciences \\ Scuola Superiore Sant'Anna \\ Piazza Martiri della Libertà 33, 56127 Pisa \\ Italy
}

Keywords: Malus domestica, hypoxia, metabolomics, gene expression, RNAseq

\begin{abstract}
Innovations in postharvest technology also deal with the modulation of gas composition in storage rooms and/or packaging, in particular concerning oxygen levels, which, for some storage protocols and fruit crops, is set at extremely low concentrations $(<1 \mathrm{kPa})$. Since the establishment of the first commercial CA rooms, the oxygen concentration showed a constant decrease throughout decades, reaching the lowest levels used in ULO (Ultra Low Oxygen), ILOS (Initial Low Oxygen Stress), and DCA (Dynamic Controlled Atmosphere) applications. This decreasing trend in oxygen concentrations used in storage rooms resulted in a general improvement of the quality parameters and the marketable life of the commodities (apples, in particular). The optimization of these techniques and the reduction of the risks (development of physiological disorders, off-odors, off-flavors) associated with keeping the fruit at such extreme conditions need to be based on a better knowledge of the metabolic responses to hypoxia. The metabolic responses of fruit tissues to low-oxygen stress are, as observed in model plants, mainly related to dramatic changes in mitochondrial respiration and the activation of the fermentative metabolism that appear to be differently affected (resulting in different levels of ethanol accumulation) by different low oxygen levels and in relation to the genetic background. Among others, ethanol, alanine, asparagine and aspartate concentrations in cortex tissue markedly change in relation to hypoxic conditions, thus representing possible metabolic markers of this kind of stress. Specific volatile compounds of apples are also differentially affected, whereas other volatile classes appear more stable. Using large-scale transcriptomic approaches coupled with metabolic profiling analyses it is now possible to better describe the global fruit responses to low oxygen conditions. In apples, in addition to the expression of genes involved in primary metabolism (major CHO, fermentation), hypoxia also affects specific secondary metabolic pathways that appear to be selectively modulated by different low oxygen treatments. Members of the ERF-VII transcription factors (TFs) gene family displayed differential expression suggesting their involvement in the modulation or controlling mechanisms of hypoxic responses, as observed in model species.
\end{abstract}

\section{INTRODUCTION}

Controlled atmosphere (CA) storage is based on reducing and increasing the $\mathrm{O}_{2}$ and $\mathrm{CO}_{2}$ concentrations, respectively that, together with the changes in the other components of the room environment (temperature, relative humidity and ethylene) and the duration of the storage have a profound effect on the physiology of harvested fruit and vegetables. Under optimal conditions (that vary depending on the species to be stored and the composition/developmental stage of the raw material), the marketable life of the commodity can be greatly extended and quality can be retained for longer time. In addition to the physiological responses to these kind of stresses/imposed conditions (that may have synergistic or antagonistic effects), the cost/benefit ratio plays a key role in the decision of applying this storage technology which requires specialized facilities and skilled human resources. This, in practice, results in a commercial use of CA limited to a 
few fruit crops; apples and European pears that can be successfully stored for several months (up to 1 year for specific apple cultivars under optimal conditions), and also kiwifruit, persimmon, pomegranate and some cultivars of Asian pears, whose storability can last up to 6 months (Kader, 1997). For specific crops such as cherries, blueberries, raspberry and strawberry storage condition based on high $(>10 \mathrm{kPa}) \mathrm{CO}_{2}$ concentrations and a more limited reduction of $\mathrm{O}_{2}$ levels can be applied (Fadanelli, 2010).

The evolution of the CA technology since its early practical applications for apple storage is characterized by a steady decrease of oxygen and increase of $\mathrm{CO}_{2}$ concentrations used in the storage rooms. In fact, the standard technology, based on an oxygen concentration of about 2-3 kPa, has, in the last 15-20 years, markedly changed and innovations are represented by CA-based methods characterized in particular by a reduction of $\mathrm{O}_{2}$ levels. One example is represented by the ultra-low oxygen (ULO) technology where $\mathrm{O}_{2}$ is maintained near $1 \mathrm{kPa}$, and ILOS (Initial Low $\mathrm{O}_{2}$ Stress) in which $\mathrm{O}_{2}$ levels are maintained as low as $0.25-0.7 \mathrm{kPa}$ for short time periods after harvest. A further step, thanks to the advances in technology that allow sensing of fruit responses to stress hypoxic stress conditions, is represented by the dynamic CA (DCA). With this technology, fruit are kept at much lower $\mathrm{O}_{2}$ concentrations than the "safe" - but not optimal - levels, but this concentration is promptly adjusted in relation to the fruit metabolic responses. Since in the DCA protocols the reduction of oxygen reaches the lowest level tolerated by the fruit (the so-called anaerobic compensation point, ACP) with a high risk of severe quality losses due to the anaerobic metabolism, the evaluation of the metabolic conditions of the fruit is crucial. The main parameters used to monitor the metabolic responses are based on the measurement of ethanol production by the fruit, the chlorophyll fluorescence and/or the Respiratory Quotient (RQ) (Schouten et al., 1997; Prange et al., 2002, 2003, 2005; Gasser et al., 2008).

Although several storage protocols for many different commodities are available today, CA-based technologies (in particular those based on extremely low concentrations of oxygen) do not always or necessarily lead to better post-storage results. As a consequence, a constant optimization of the product's response to CA is needed. For this goal, the metabolic responses and molecular regulation of fruit tissues to such conditions are needed to be further characterized based on new research tools and integrated approaches.

\section{LOW-OXYGEN STORAGE AND FRUIT QUALITY}

Generally speaking, the decrease in oxygen concentration for the long-term storage of some apple and pear cultivars coupled with the development of technical advancements and appropriate monitoring systems, has in general led to a better and prolonged maintenance of quality parameters and a longer marketable life. This is mainly due to a reduction of the respiratory activity of the product and a general decrease of metabolism. Early investigations (Lavilla et al., 1999) on the effects of oxygen concentration decrease from 3 to $1 \%$ found that the lowest concentration (ULO) showed the highest ability to maintain apple ('Granny Smith') firmness after 7 months of storage. The effects on quality parameters induced by the further reduction of oxygen concentration characterizing the DCA protocols have been studied in different apple cultivars. In 'Elstar' firmness and color retention was greater than in ULO-stored fruits (Veltman et al., 2003) and fruit of 'Golden Delicious', 'Jonagold' and 'MacIntosh' were firmer during 9 months storage in DCA than in CA (DeLong et al., 2004). Compared to the ULO technique, better scores for crispness and acidity have been detected in 'Elstar' (Schouten et al., 1997) and for firmness in 'Golden Delicious' (Zanella et al., 2008). Not always DCA results in improvements or better quality maintenance compared to ULO storage. This is the case of fruit of 'Gala' and 'Fuji' where $\mathrm{O}_{2}$ levels lower than $1 \mathrm{kPa}$ did not improve consistently firmness compared to optimal ULO-CA (Zanella et al., 2008).

In addition to organoleptic/sensorial parameters, hypoxic conditions can exert other important positive effects during storage life, such as the control of superficial scald or the prevention of many senescence-related physiological disorders in apples and pears. 
Superficial scald is a symptom of chilling injury and is one of the most common postharvest physiological disorders in pome fruit. Typical symptoms are brown or black patches on the fruit skin that appear during or after storage (Zanella, 2003; Lurie and Watkins, 2012). The effects of low oxygen atmospheres on superficial scald have been described (Wang and Dilley, 1999) and successful control of scald in susceptible apple cultivars can be achieved by means of ILOS for short storage term (Truter et al., 1994). Research on storage at very low oxygen levels in DCA demonstrated the efficient control of scald on different cultivars for several seasons, even after long term storage (Zanella et al., 2005; Zanella and Sturz, 2013). It has been postulated that the prevention of scald development, that is assumed to be an oxidative process, might involve production of anaerobic metabolites (such as ethanol) induced by hypoxic conditions, causing a delay in ethylene and $\alpha$-farnesene biosynthesis and oxidation (Ghahramani and Scott, 1998; Sabban-Amin et al., 2011). Pre-storage treatments (10 d) with low-oxygen atmosphere $(0.5 \%)$ are also effective in reducing superficial scald on 'Granny Smith' apples, after six months of cold storage at $0^{\circ} \mathrm{C}$ plus seven days at $20^{\circ} \mathrm{C}$ and this has been associated with a reduced ethylene biosynthesis, and less production of $\alpha$-farnesene and its oxidation product, 6-methyl-5-hepten-2-one (MHO) (Pesis et al., 2014).

The reduction of oxygen levels at concentrations close to ACP for periods longer than few days is a stress condition for the harvested product and has a profound impact on fruit metabolism. The optimization of techniques based on prolonged storage at extremely low oygen concentrations must be based on the elucidation of the hypoxia induced/ affected processes, thus exploiting the beneficial effects of the reduced metabolism and reducing the risks of the appearance of low oxygen-associated physiological disorders and the decrease of quality parameters. Disorders occurring during long-term storage of apples and pears under hypoxia cause economic loss and consumer dissatisfaction considering that disordered fruit cannot be distinguished externally from sound fruit (Franck et al., 2007). One of the most important and frequent physiological disorders associated with long-term low oxygen storage is browning of the flesh. This is the result of a metabolic unbalance and major changes in respiration. Lee et al. (2012) reported that in 'Braeburn' apples stored under controlled atmosphere, flesh browning is associated with increased fermentative metabolic products such as acetaldheyde and ethanol. Vandendriessche et al. (2013) demonstrated, by means of NMR analysis that, in the same apple cultivar, two different storage conditions (optimal CA and brown-inducing CA) caused significant changes in metabolite levels, in particular concerning pyruvate, citrate, fumarate, alanine, chlorogante, metahnol, ethanol, acethaldehyde, thus confirming that the onset of the physiological disorder is related to altered primary metabolism. Brown tissue in stored pears was clearly characterized by a decrease of malic acid and an increase in fumaric acid and gamma aminobutyric acid (GABA), which indicate a reduced metabolic activity at the level of the Krebs cycle and a putative block of the GABA shunt pathway (Pedreschi et al., 2009). GABA and gluconic acid have been shown to be metabolic markers for core breakdown.

An important effect of low oxygen storage of fruit is also related to the production of volatile organic compounds (VOCs). It has been reported that CA-stored apples produce significantly less volatile compounds during shelf-life than those stored under low temperature with natural air (Plotto et al., 1999; López et al., 2007). Considering the responses to extremely low concentrations of 'Royal Gala' apples, decreasing oxygen concentation from 1.0 to $0.5 \mathrm{kPa}$ for 8 months appeared to affect mainly the straight-chain esters (that decreased) while branched-chain ester production did not change (Both et al., 2014). The long-term storage under extreme low-oxygen concentrations and the induced changes in metabolism (e.g., the activation of anaerobic reactions) may lead to the development of off-odors. Chemical compounds that cause the mouldy, musty and earthy off-odours of apple fruit stored under controlled atmosphere at low temperature for several months have been recently reported by Kaack (2014). Five peaks associated with oxidation products of $\alpha$-farnesene seemed to be responsible for the off-odors. In 'Scarlett Spur Red Delicious' apples, volatile production differed quantitatively in relation to 
oxygen pressure, acetaldehyde, ethanol, and ethyl ester accumulation increased with decreased oxygen supplying during the first weeks of storage (Lumpkin et al., 2014). Differences in volatile accumulation were evident through six months of the above described storage protocols. The rate of ethanol accumulation increased with decreased oxygen concentration whereas $\alpha$-farnesene was not detected throughout the storage period.

\section{MOLECULAR RESPONSES TO LOW OXYGEN}

As above described, the application of low oxygen storage protocols has shown to be extremely beneficial for preserving the quality of harvested fruit products but these methods, when not appropriately applied (gas concentrations, storage duration, fruit physiological conditions) and monitored, may result in the development and appearance of disorder symptoms and negative attributes result of the activation of hypoxia-related metabolism. It is well known that fruit responses to modified $\mathrm{O}_{2}$ levels include changes at the levels of primary and secondary metabolism (Kader, 1997). However, despite many commercial applications of hypoxic treatments, the precise mode of action of low oxygen in fruit tissues and ripening at molecular level is not well understood.

Decline in oxygen availability (hypoxia) triggers changes in gene transcription and messenger RNA translation that promote anaerobic metabolism. During the last ten years characterization of the molecular elements of low oxygen sensing and signaling has grown extensively in model species. Efforts of research community led to characterization of two main sensing mechanisms in plant organisms: direct and indirect (Gibbs et al., 2011; Licausi et al., 2011, 2012; Bailey-Serres et al., 2012). First breakthrough was done on elucidating molecular basis of rice (Oryza sativa) tolerance to submergence. Direct low oxygen sensor was identified in Arabidopsis thaliana, where a conserved $N$-terminal motif on some Ethylene Response Factors $(E R F S)$ group VII transcription factor family targets the fate of the protein under normoxia/hypoxia. Taken together, these studies suggest that species-specific modes of action to respond to lowoxygen stress evolved from the same basic genetic elements. Indirect oxygen-sensing mechanisms could be based on low energy status, variations in carbohydrate availability, reactive oxygen species (ROS) signals, nitric oxide (NO)-related responses, NAD/NADH ratio, calcium fluxes and homeostatic reactions to intracellular $\mathrm{pH}$ changes (Banti et al., 2013).

In transcriptomic studies, a core of about 50 genes that respond ubiquitously to oxygen deficiency has been identified by microarray analyses in several plant species (Mustroph et al., 2010). This includes genes involved in fermentative and alanine metabolism, scavenging and detoxification of reactive oxygen and nitrogen species as well as a big number of proteins of unknown function. A specific comparison of the transcriptional responses to hypoxic conditions in rice and Arabidopsis pointed out that the common genes upregulated were largerly enriched in photosynthesis, major CHO metabolism and protein degradation functions, whereas the common genes downregulated under anaerobic conditions were largerly enriched in cell wall and secondary metabolism functions (Narsai et al., 2011). An analysis of microarray data from experiments on Arabidopsis held under different hypoxic conditions and aimed at searching for the genes related to oxidative stress response revealed the intrinsic connection between oxygen deprivation stress and the increased expression of genes coding for oxygenases (e.g., Fedependent ketoglutarate oxygenase, ACC-oxidase, desaturases, alternative oxidase, etc.) (Blokhina et al., 2014).

These studies have been performed on model species based on analyses of the responses of vegetative tissues where most of the molecular studies related to anoxia/hyopxia conditions have been carried out (van Dongen and Licausi, 2014). The effects of low-oxygen conditions on molecular aspects and gene expression regulation in fleshy fruit have been scarcely studied up to now. Early data provided by Kanellis et al. (1991) indicate that also in fruit tissues (avocado) cell wall metabolism is affected by postharvest low oxygen stress: in fact, a marked reduction of cellulase (EGase) mRNA 
accumulation was observed in fruit held for 3 days at oxygen concentrations lower than $7.5 \%$. The transcription of a specific arabinogalactan protein $(A G P)$ gene, a glycosylated member of the superfamily of hydroxyproline rich glycoproteins (HRGPs), is induced in tomato fruit by several stressors, including anoxia and hypoxia (Fragkostefanakis et al., 2012). This information reinforces the hypothesis that low oxygen conditions affect fruit ripening in general, and the softening process in particular, by modulating the expression of specific cell-wall related genes. Genes involved in major $\mathrm{CHO}$ metabolism appear to be affected by postharvest low-oxygen stress also in fruit tissues: following a 3-day postharvest anoxic treatment, genes encoding sucrose synthase $(S S)$, neutral invertase $(N I)$, sucrose-P-synthase $(S P S)$, and sorbitol dehydrogenase $(S D H)$ resulted up-regulated in peach fruit (Lara et al., 2011). Sucrose synthase was discovered as one of the major anaerobic polypeptides in maize and its expression is induced under oxygen deficiency in many species (Mustroph et al., 2014). RNAseq data of 'Granny Smith' apples stored for 28 days at 0.4 and $0.8 \mathrm{kPa}$ oxygen concentrations revealed an increased expression of $S S$ in both samples (Cukrov et al., unpublished). The upregulation of SS under extreme lowoxygen conditions would indicate that also in apple fruit a shift from invertase to sucrose synthase occurs under hypoxia suggesting, as observed in model species, the activation of alternative pathways that can use inorganic pyrophosphate (PPi) instead of ATP for phosphorylation reactions. In addition to $S S$, a number of genes defined as hypoxiarelated in studies performed on model species, resulted differentially expressed in 'Granny Smith' apples held under low-oxygen stress: among these, oxygenases (ACO, Fe-dependent ketoglutarate oxygenase), genes involved in major $\mathrm{CHO}$ metabolism (SS, but also fructokinase, hexokinase) and in fermentation (alcohol dehydrogenase, $A D H$ and pyruvate decarboxylase, PDC) were detected.

In plants, lack of oxygen causes a reduction in respiratory efficiency and, as a consequence, in energy production (Gupta et al., 2009). Therefore, under hypoxia/anoxia ATP synthesis is mostly provided by glycolysis coupled with NAD regenerative pathways, including ethanolic fermentation and alanine production (Ismond et al., 2003; Ricoult et al., 2006). This metabolic reset seems to occur also in fruit tissues: accumulation of $A D H$ transcripts were detected in avocado fruit held under oxygen concentrations ranging between 0 and 5\% (Loulakakis et al., 2006), and two $A D H$ (as well as two $P D C$ ) genes resulted, based on RNAseq data, highly induced in 'Granny Smith' apples after 4 weeks storage at 0.4 and $0.8 \mathrm{kPa}$ oxygen concentrations (Cukrov et al., unpublished). Interestingly, these two levels of low-oxygen stress induced different accumulation of ethanol, and, with less pronounced difference between the two treatments, of lactate, aspartate, alanine, asparagine and uridine (Brizzolara et al., unpublished). This would indicate that a slight difference of oxygen concentration has a marked effect in modulating specific pathways of apple fruit metabolism. In this context a possible role of specific Transcription Factors (TF) can be postulated.

Recent discoveries have highlighted that, in Arabidopsis, transcriptional adjustments to oxygen depletion are triggered by group VII Ethylene Response Factors (ERF) that, being involved in an oxygen-dependent proteolytic mechanism ( $\mathrm{N}$-end rule pathway), act like a bridge directly linking oxygen sensing and regulation of low oxygenresponsive genes (Gibbs et al., 2011; Licausi et al., 2011). At least two members of the apple group VII $E R F$ gene family show a marked up-regulation when 'Granny Smith' fruit are stored und low-oxygen conditions $(0.4$ and $0.8 \mathrm{kPa})$, with the highest level of transcript accumulation occurring in correspondence of the lowest oxygen concentration (Cukrov et al., unpublished data). These data coupled with preliminary western blot analysis showing different specific ERF protein accumulation in normoxic and hypoxic apple cortex samples (Zemiani and Ruperti, pers. commun.) suggest that the regulatory mechanisms of the transcriptional reconfiguration taking place under oxygen deficiency are similar in vegetative tissues (of model species) and fleshy fruit.

Literature Cited

Bailey-Serres, J., Fukao, T., Gibbs, D.J., Holdsworth, M.J., Lee, S.C., Licausi, F., Perata, 
P., Voesenek, L.A.C.J. and van Dongen, J.T. 2012. Making sense of low oxygen sensing. Trends Plant Sci. 17:1-10.

Banti, V., Giuntoli, B., Gonzali, S., Loreti, E., Magneschi, L., Novi, G., Paparelli, E., Parlanti, S., Pucciariello, C., Santaniello, A. And Perata, P. 2013. Low oxygen response mechanisms in green organisms. Int. J. Mol. Sci. 14:4734-4761.

Blokhina, O.B., Toronen, P. and Fagerstedt, K.V. 2014. Oxidative stress components explored in anoxic and hpoxic global gene expression data. p.19-39. In: J.T. van Dongen and F. Licausi (eds.), Low-Oxygen Stress in Plants. Springer.

Both, V., Brackmann, A., Thewes, F.R., de Freita Ferreira, D. and Wagner, R. 2014. Effect of storage under extremely low oxygen on the volatile composition of 'Royal Gala' apples. Food Chem. 156:50-57.

DeLong, J.M., Prange, R.K., Leyte, J.C. and Harrison, P.A. 2004. A new technology that determines low-oxygen threshold in controlled-atmosphere-stored apples. HortTechnology 14:262-266.

Fadanelli, F. 2010. Stato attuale delle applicazioni dell'atmosfera controllata in Italia. Frutticoltura 11:2-7.

Fragkostefanakis, S., Dandachi, F. and Kalaitzis, P. 2012. Expression of arabinogalactan proteins during tomato fruit ripening and in response to mechanical wounding, hypoxia and anoxia. Plant Physiol. Biochem. 52:112-118.

Franck, C, Lammertyn, J., Ho, Q.T., Verboven, P., Verlinden, B. and Nicolai, B.M. 2007. Browning disorders in pear: a review. Postharvest Biol. Technol. 43:1-13.

Gasser, F., Eppler, T., Naunheim, W., Gabioud, S. and Hoehn, E. 2008. Control of the critical oxygen level during dynamic CA storage of apples by monitoring respiration as well as chlorophyll fluorescence. Acta Hort. 796:69-76.

Ghahramani, F. and Scott, K.J. 1998. Oxygen stress of Granny Smith apples in relation to superficial scald, ethanol, $\alpha$-farnesene and conjugated trienes. Aust. J. Agric. Res. 49:207-210.

Gibbs, D.J.D., Lee, S.C.S., Isa, N.M.N., Gramuglia, S.S., Fukao, T.T., Bassel, G.W.G., Correia, C.S.C. et al. 2011. Homeostatic response to hypoxia is regulated by the Nend rule pathway in plants. Nature 479:415-418.

Gupta, K.J., Zabalza, A. and van Dongen, J.T. 2009. Regulation of respiration when the oxygen availability changes. Physiol. Plant. 137:383-391.

Ismond, K.P., Dolferus, R., De Pauw, M., Dennis, E.S. and Good, A.G. 2003. Enhanced low oxygen survival in Arabidopsis through increased metabolic flux in the fermentative pathway. Plant Physiol. 132:1292-1302.

Kaack, K. 2014. Mouldy, musty earthy off-odour of apple fruits. Hort. Sci. (Prague) 41:44-47.

Kanellis, A.K., Solomos, T. and Roubelakis-Angelakis, K.A. 1991. Suppression of cellulase and polygalacturonase and induction of alcohol dehydrogenase isoenzymes in avocado fruit mesocarp subjected to low oxygen stress. Plant Physiol. 96:269-274.

Lara, M.V., Budde, C.O., Porrini, L., Borsani, J., Murray, R., Andreo, C.S. and Drincovich, M.F. 2011. Peach (Prunus persica) fruit response to anoxia: reversible ripening delay and biochemical changes. Plant Cell Physiol. 52:392-403.

Lavilla, T., Puv, J., Lopez, M.L., Recasens, I. and Vendrell, M. 1999. Relationships between volatile production, fruit quality, and sensory evaluation in Granny Smith apples stored in different controlled-atmosphere treatments by means of multivariate analysis. J. Agric. Food Chem. 47:3791-3803.

Licausi, F. 2012. Molecular elements of low-oxygen signaling in plants. Physiologia Planta 148:1-8.

Licausi, F., Kosmacz, M., Weits, D.A., Giuntoli, B., Giorgi, F.M., Voesenek, L.A.C.J., Perata, P. et al. 2011. Oxygen sensing in plants is mediated by an $\mathrm{N}$-end rule pathway for protein destabilization. Nature 479:419-422.

Lee, J., Mattheis, J.P. and Rudell, D.R. 2012. Antioxidant treatment alters metabolism associated with internal browning in 'Braeburn' apples during controlled atmosphere storage. Postharvest Biol. Technol. 68:32-42. 
López, M.L., Villatoro, C., Fuentes, T., Graell, J., Lara, I. and Echeverría, G. 2007. Volatile compounds, quality parameters and consumer acceptance of 'Pink Lady' apples stored in different conditions. Postharvest Biol. Technol. 43:55-66.

Loulakakis, C.A., Hassan, M., Gerasopoulos, D. and Kanellis, A.K. 2006. Effects of low oxygen on in vitro translation products of poly $(\mathrm{A})+$ RNA, cellulase and alcohol dehydrogenase expression in preclimacteric and ripening-initiated avocado fruit. Postharvest Biol. Technol. 39:29-37.

Lumpkin, C., Fellman, J.K., Rudell, D.R. and Mattheis, J. 2014. 'Scarlett Spur Red Delicious' apple volatile production accompanying physiological disorder development during low $\mathrm{pO}_{2}$ controlled atmosphere storage. J. Agric. Food Chem. 62:1741-1754.

Lurie, S. and Watkins, C.B. 2012. Superficial scald, its etiology and control. Postharvest Biol. Technol. 65:44-60.

Mustroph, A., Hess, N. and Sasidharan, R. 2014. Hypoxic energy metabolism and PPi as an alternative energy currency. p.165-184. In: J.T. van Dongen and F. Licausi (eds.), Low-Oxygen Stress in Plants. Springer.

Mustroph, A., Lee, S.C., Oosumi, T., Zanetti, M.E., Yang, H., Ma, K. et al. 2010. Crosskingdom comparison of transcriptomic adjustments to low-oxygen stress highlights conserved and plant-specific responses. Plant Physiol. 152:1484-1500.

Narsai, R., Rocha, M., Geigenberger, P., Whelan, J. and van Dongen, J.T. 2011. Comparative analysis between plant species of transcriptional and metabolic responses to hypoxia. New Phytol. 190:472-487.

Pedreschi, R., Hertoog, M., Robben, J., Lilley, K. et al. 2009. Gel-based proteomics approach to the study of metabolic changes in pear tissue during Storage. J. Agric. Food Chem. 57:6997-7004.

Pesis, E., Feygenberg, O., Sabban-Amin, R., Ebeler, S.E., Mitcham, E.J. and Ben-Arie, R. 2014. Low oxygen pre-storage treatment is effective in reducing chilling injuries of deciduous fruit. Int. J. Postharvest Technol. Innov. 4:23-32.

Plotto, A., Mc Daniel, M.R. and Mattheis, J.P. 1999. Characterisation of 'Gala' apple aroma and flavour: differences between controlled atmosphere and air storage. J. Amer. Soc. Hortic. Sci. 124:416-423.

Prange, R., DeLong, J. and Harrison, P.A. 2005. Quality management through respiration control: is there a relationship between lowest acceptable respiration, chlorophyll fluorescence and cytoplasmic acidosis? Acta Hort. 682:823-830.

Prange, R., DeLong, J., Harrison, P., Leyte, J. and McLean, S.D. 2003. Oxygen concentration affects chlorophyll fluorescence in chlorophyll-containing fruit and vegetables. J. Amer. Soc. Hort. Sci. 128:603-607.

Prange, R., DeLong, J., Leyte, J. and Harrison, P. 2002. Oxygen concentration affects chlorophyll fluorescence in chlorophyll-containing fruit. Postharvest Biol. Technol. 24:201-205.

Ricoult, C., Orcaray Echeverria, L., Cliquet, J.B. and Limami, A.M. 2006. Characterization of alanine aminotransferase (AlaAT) multigene family and hypoxic response in young seedlings of the model legume Medicago truncatula. J. Exp. Bot. 57:3079-3089.

Sabban-Amin, R., Feygenberg, O., Belausov, E. and Pesis, E. 2011. Low oxygen and 1MCP pretreatments delay superficial scald development by reducing reactive oxygen species (ROS) accumulation in stored 'Granny Smith' apples. Postharvest Biol. Technol. 62:295-304.

Schouten, S.P., Prange, R., Verschoor, J.A., Lammers, T.R. and Oosterhaven, J. 1997. Improvement of quality of 'Elstar' apples by dynamic control of ULO conditions. Proc. $7^{\text {th }}$ Intl. Controlled Atmosphere Res. Conf., Davis, Calif. 2:71-78.

Truter, A.B., Combrink, J.C. and Burger, S.A. 1994. Control of superficial scald in 'Granny Smith' apples by ultra-low and stress levels of oxygen as an alternative to diphenylamine. Hort. Sci. 69:581-587.

Vandendriessche, T., Schaffer, H., Verlinden, B.E., Humpfer, E., Hertog, M.L.A.T.M., 
Nicolai, B.M. 2013. High-throughput NMR based metabolic profiling of Braeburn apple in relation to inernal browning. Postharvest Biol. Technol. 80:18-24.

van Dongen, J.T. and Licausi, F. (eds.). 2014. Low-Oxygen Stress in Plants. Springer.

Veltman, R.H., Verschoor, J.A. and van Dugteren, J.H.R. 2003. Dynamic control system (DCS) for apples (Malus domestica Borkh. Cv. Elstar): optimal quality through storage based on product response. Postharv. Biol. Technol. 27:79-86.

Wang, Z. and Dilley, D.R. 1999. Control of superficial scald of apples by low-oxygen atmospheres. HortScience 34:1145-1151.

Zanella, A. 2003. Control of apple superficial scald and ripening - a comparison between 1-methylcyclopropene and diphenylamine postharvest treatments, initial low oxygen stress and ultra low oxygen storage. Postharvest Biol. Technol. 27:69-78.

Zanella, A., Cazzanelli, P. and Rossi, O. 2008. Dynamic controlled atmosphere (DCA) storage by the means of chlorophyll fluorescence response for firmness retention in apple. Acta Hort. 796:77-82.

Zanella, A. and Stürz, S. 2013. Replacing DPA postharvest treatment by strategical application of novel storage technologies controls scald in 1/10th of EU's apples producing area. Acta Hort. 1012:419-426. 